\title{
Models of the Effects of Dose Heterogeneity and Escape on Selection Pressure for Pesticide Resistance
}

\author{
M. W. Shaw
}

Department of Agricultural Botany, School of Plant Sciences, The University of Reading, 2 Earley Gate, Whiteknights, Reading RG6 6AU, U.K. Accepted for publication 14 December 1999.

\begin{abstract}
Shaw, M. W. 2000. Models of the effects of dose heterogeneity and escape on selection pressure for pesticide resistance. Phytopathology 90:333339.

Models of the intensity of selection for resistance resulting from the application of heterogeneous doses of pesticide are developed. Dose heterogeneity is assumed to arise from two factors. First, a proportion of the population completely escapes treatment. Second, the rest of the population receives a distribution of doses that can be described by a probability distribution that is approximately logistic on a logarithmic scale. Various assumptions about the biology of the target population are

explored, including both sexual and asexual reproduction of haploid and diploid organisms with either polygenic or monogenic inheritance of resistance. There are two major conclusions. (i) The presence of escape produces a maximum in the graph of selection intensity against dose or control; below the dose corresponding to the maximum, selection is reduced if dose is reduced, but above the maximum, selection is reduced if dose is increased. Informal arguments suggest it is unlikely that field doses would often be above the maximum dose, so selection would usually be decreased by decreasing dose. (ii) For the same control, selection for resistance is reduced by greater heterogeneity of pesticide dose, even though larger average doses may be needed.
\end{abstract}

Pesticide application techniques are imperfect, and pesticides themselves are mobile in and on plants. The dose consumed or absorbed by individuals of the target species, therefore, usually is variable. Most models of the evolution of pesticide resistance have approximated this variability by dividing the target population into just two categories: treated and untreated $(2,4,9,12-16$, $18,21,23,24)$. These models show that the untreated proportion of the population may greatly alter the rate at which resistance to a pesticide develops, if suitable variation is present in the population. For convenience, I will refer to the existence of an untreated portion of the population as "escape." In this paper, I will show that increased variation in dose received may substantially slow down the development of resistance, even when the resulting control is unchanged or in the absence of escape. Also, I will draw attention to the existence, if there is escape, of intermediate doses that maximize the rate at which resistance develops.

When a pesticide is applied to a target population in the field, the biological effect of the dose received varies between individuals for several reasons. Individuals themselves differ in their sensitivity; their behavior or growth form vary in ways that affect the amount of pesticide individuals absorb; pesticides may be diluted, concentrated, or fractionated in their interactions with plants and the environment; and the spatial distribution of the original application is never even.

Standard advice on pesticide application is to maintain "full" doses to avoid the development of resistance (1). The rationale for this has been stated clearly for diploid organisms with resistance controlled by a single locus and in which heterozygotes are less resistant than the resistant homozygote $(13,24)$. In these cases, the heterozygotes will be effectively susceptible if a large enough dose is applied, and the variation in the population on which selection can act will be vastly reduced. However, this argument

Corresponding author: M. W. Shaw; E-mail address: m.w.shaw@reading.ac.uk

Publication no. P-2000-0120-01R

(c) 2000 The American Phytopathological Society involves the assumption that individuals in a population receive a single dose, as in a laboratory bioassay. In reality, the actual dose received will be very variable, as argued above, and the consequences of varying the average dose applied in such a case are not obvious.

In other cases, such as clonal or haploid organisms or oligogenically or polygenically controlled resistance, the argument that full doses will retard the emergence of resistance is less clear and rests on two assumptions: (i) at reduced doses, there will be more survivors, and those survivors will tend to carry more resistant alleles or be from more resistant clones than the average before treatment; and (ii) a constant proportion of the population completely escapes treatment, and it is mainly from this that the population is refounded. However, even with the underlying assumption that all individuals experience a single dose, the argument is incomplete, because at higher doses there will be fewer survivors but they would be expected to be more resistant.

Gardner et al. (7) present a very clear exposition of this argument. They point out that extreme individuals-expected perhaps at frequencies of 1 in $10^{20}$-actually will be absent in any real, finite population, so high doses can be arranged to completely kill a partially resistant population. It is implicit that the population then will be refounded by immigrants from never-treated refugia. Without the second assumption from the previous paragraph, the pest problem would simply disappear. If the kill level in the treated population was less than $100 \%$, it is hard to understand why the extent of selection would not increase with increasing dose.

However, it may be hard to guarantee absolutely complete coverage of the previously treated population, especially with mobile organisms, such as insects or fungi. The evolutionary outcome then depends on the quantitative detail of the outcome of mixing fewer but more resistant individuals with the escapees. Modeling can help in establishing a framework in which to understand the outcome of experiments.

It is well established that spray application of pesticides is an imperfect technique. The aim is usually to obtain even coverage of foliage, but this is very difficult, even with air-assisted sprayers, controlled droplet sizes, or electrical charging of spray drops (3, 
$6,8,11)$. It is useful, for the models that follow, to try to obtain quantitative estimates of the variation, after a spray, in the amount of chemical applied. The scale of interest is roughly the portion of the plant from which a pathogen or pest might absorb a pesticidein many cases, much less than $1 \mathrm{~cm}^{2}$.

Estimates of variation in dose applied to single leaves are given in Cross and Berrie (3), Koch and Spieles (10), and Koch and Weisser (11). These estimates suggest a coefficient of variation of the order of 1 and distributions that are strongly skewed and better approximated by a log-normal than a normal distribution. Here, we are interested in the variation in doses to individuals, so the coefficient of variation that is relevant is that which measures the variation between regions that are the size of one individual, over a region within which the population of interest is freely mixing. This increases the variance over that observed between leaves within one zone of foliage in two ways. As the scale of interest decreases, variance would be expected to rise, because less and less of the foliage is averaged over. At the other end of the scale of interest, extra variance would arise because sprays do not penetrate a canopy evenly, nozzles differ, etc. This suggests that coefficients of variation might easily reach orders of 2 or more.

Even if a spray were deposited evenly on a plant, the dose is not static. Systemic chemicals are redistributed within the plant, often to a substantial extent, and may become concentrated as well as diffusing into more even doses. For example, Metcalfe et al. (17) published data showing how the triazole fungicide flutriafol, under laboratory conditions, became concentrated at the tips of leaves, producing different selection patterns between two isolates of differing sensitivity according to the time at which they infected or reproduced and depending on position on the leaf.

The exact probability distribution of dose applied to a target population in a real setting is unknown and would be very hard to determine. However, it is likely that many factors combine to determine the dose experienced by any individual and that these factors combine multiplicatively rather than additively. For example, being at the top of a cereal crop rather than in the interior might double the risk of receiving a large dose, while being small might halve it (additive combination would imply instead that being small changes the likely dose by a defined amount: so many extra micrograms when at the top, etc.). If the variation in dose received arises from many multiplicative factors, then the central limit theorem applies, and the resulting probability distribution should converge on a log-normal distribution, in which the logarithm of the dose received is distributed normally. In practice, the approximation to a normal distribution may not be very good; in particular, there are many cases of approximately normal distributions in which the probability of extreme events is much larger

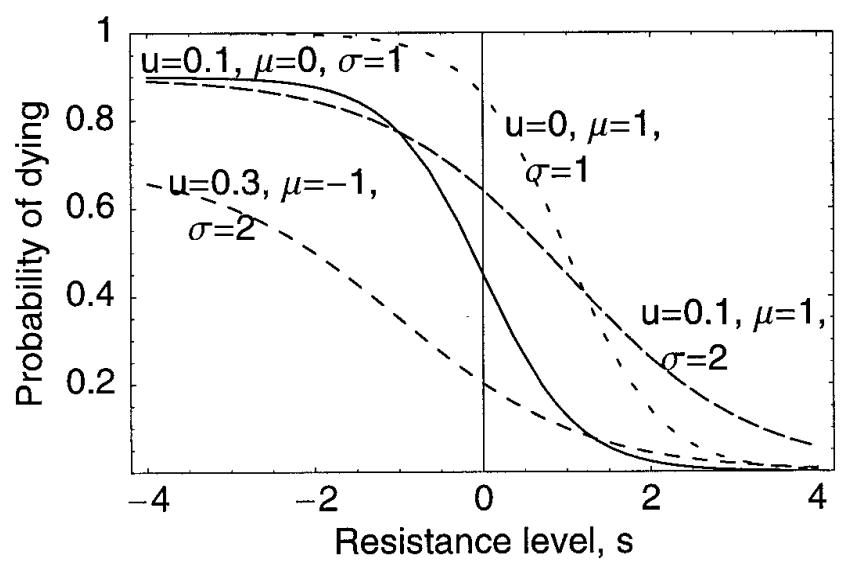

Fig. 1. The probability of an individual dying after pesticide treatment, as a function of its intrinsic sensitivity level, as given in equation 2. Four parameter combinations are shown. than suggested by the normal distribution. However, a bell-shaped probability distribution of the logarithm of dose is plausible. The logistic distribution, introduced in the next section, is close to the normal distribution in the center but specifies greater probabilities of extreme events. There might or might not be, in addition, a distinct category of untreated individuals that makes the distribution bimodal.

The aim of the rest of this paper is to model the effect of variation in dose on the selective force applied to a population. Both polygenic and monogenic inheritance patterns for resistance will be considered. The models do not mimic a specific real system, but the arguments applied allow fairly general qualitative conclusions to be drawn, which it should be possible to test in a variety of settings. In particular, resistance management might suggest obtaining a required level of control using larger doses applied in a more variable fashion. The arguments also apply to selection of more resistant individuals. It seems possible, however, that the arguments also may apply to some of the side-effects of pesticide applications on nontarget parts of the ecosystem, which is the opposite of the accepted practice for minimizing the harmful sideeffects of pesticides.

\section{THEORY AND APPROACHES}

All the models described below make the same assumption about the probability distribution of the effective log-dose of pesticide encountered by an individual. It is assumed that a proportion, $u$, of the population completely escapes the pesticide, and the remainder receives a log-dose logistically distributed around a mean, $\mu_{d}$, with a standard deviation, $\sigma_{d}$, i.e., if $X$ is the log-dose received, the probability, $\operatorname{Pr}\{X<x\}$, that the dose received is less than a given value, $x$, is specified by a function:

$$
P(x)=u+\frac{(1-u)}{1+\exp \left(-\frac{\pi}{\sqrt{3}} \frac{\left(x-\mu_{d}\right)}{\sigma_{d}}\right)}
$$

The logistic distribution is a symmetric, bell-shaped distribution similar to the normal distribution but with more probability at extreme values. The logistic distribution is easier to work with because the distribution function can be written down explicitly instead of in the form of an integral that must be evaluated numerically. As argued above, the longer tails of the distribution are more realistic than the very restricted range of the normal distribution. The sensitivity of the models to this assumption is discussed later.

Because log-dose is the relevant variable that describes the effectiveness of the dose received, $\sigma_{d}$ is given approximately by the

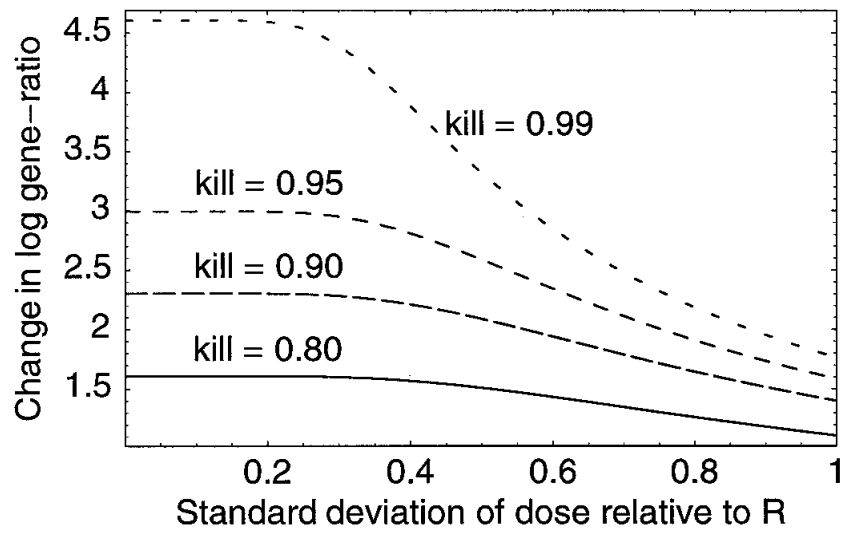

Fig. 2. The change in the log-ratio of sensitive to resistant genotypes, $\Theta$, after a single application of a pesticide as a function of spread in dose, $\sigma_{d}$, for various values of control. Escape $(u)$ is 0 for all lines. $R=$ level of resistance of the more resistance form. 
coefficient of variation of distributions of untransformed doses. (This can be demonstrated by treating the log-dose as a function of dose, making a Taylor expansion of log-dose around the mean and calculating the variance of the log-dose using this expansion.)

The sensitivity of an individual to a pesticide at the time of encounter is assumed to be described by a number, $s$, that varies between individuals. If an individual with sensitivity $s$ encounters a dose of $s$ or more, it will die; otherwise, it will live. This is the assumption underlying probit analysis of dose-effect curves, except that here $s$ is assumed to be logistically rather than normally distributed. The probability that an individual with sensitivity $s$ will survive is the same as the probability that it will encounter a dose less than $s$. This probability is given by equation 1 , so the probability that an individual will die is the complement of equation 1 but applied to variable $s$. With a little rearrangement the probability becomes

$$
P(s)=\frac{(1-u)}{1+\exp \left(\frac{\pi}{\sqrt{3}} \frac{\left(s-\mu_{d}\right)}{\sigma_{d}}\right)}
$$

Figure 1 shows the shape of this relationship for a few specific parameter sets. A more general formula would be that $s$ is the mean value of sensitivity for an individual, and the actual outcome after encountering a pesticide is determined by some probability distribution depending on $s$ and $x$. The probability of death then would be a function, $g(s, x): g$ might represent, for example, a normal distribution depending on $s-x$. The overall probability of death after pesticide application would be the convolution of equation 1 and $g$ with respect to $x$. In the special case in which the variation of an individual's sensitivity to $s$ is slight relative to variation in the dose received, $x$, a Dirac delta function (i.e. a thin spike), will adequately describe $g$, leading to equation 2. Qualitatively, such variation amounts to reduced heritability of the trait. This does not qualitatively affect the arguments that follow. Therefore, only the simpler form of equation 2 is used in the rest of the paper.

Resistance inherited as a single trait. In the case of resistance inherited as a single trait, there are two qualitatively distinct resistance phenotypes corresponding to two peaks in the plot of the probability density function of sensitivity. The distance between the peaks is assumed to be much greater than variation in sensitivity around the two major peaks, so an individual is unambiguously resistant or sensitive depending on the alleles at a single genetic locus.

Nonoverlapping generations in a haploid or clonal organism. Nonoverlapping generations in a haploid or clonal organism can be analyzed by determining the change in the ratio of resistant to susceptible alleles after a single generation of selection. It is convenient to adjust the dose scale by adding or subtracting a constant

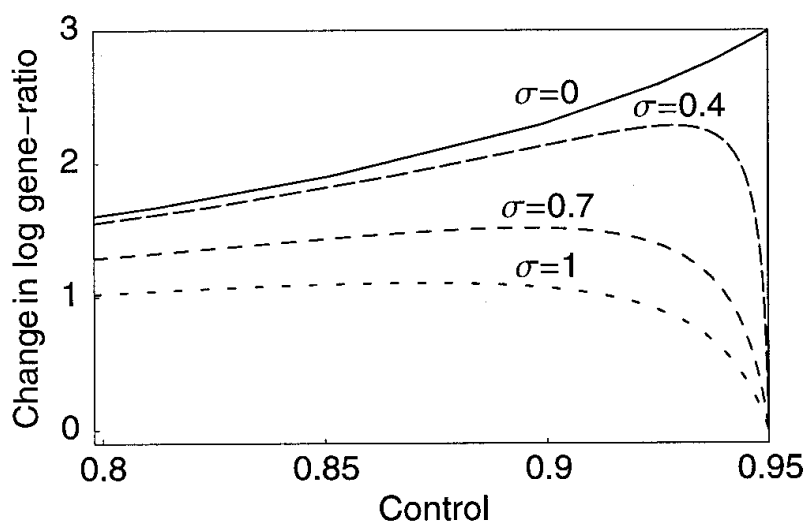

Fig. 3. The change in the log-ratio of sensitive to resistant genotypes, $\Theta$, and control, for different values of standard deviation in dose received, $\sigma_{d}$, with escape fixed at $5 \%$ for all lines. so that the more sensitive genotype has a resistance of 0 . The more resistant form can be defined as having a resistance of $R$ on this scale. If the initial proportion of sensitive genotypes is $p$ and the total relative survival is $\bar{w}$, then after treatment, equation 1 can be used to show that the new proportion of the sensitive genotype is given by

$$
p^{\prime}=\frac{p}{\bar{w}}\left[u+\frac{(1-u)}{1+\exp \left(\pi \mu_{d} / \sqrt{3} \sigma_{d}\right)}\right]
$$

and the new proportion of the resistant form is given by

$$
1-p^{\prime}=\frac{(1-p)}{\bar{w}}\left\{u+\frac{(1-u)}{1+\exp \left[\left(\pi / \sqrt{3} \sigma_{d}\right)\left(\mu_{d}-R\right)\right]}\right\}
$$

The new gene ratio, $p^{\prime} /\left(1-p^{\prime}\right)$, is the ratio of equations 3 and 4 . A useful measure of the strength of selection is the logarithm of the ratio of the new to the old gene ratio, $\Theta$. $R$ appears in equation 4 only as the dimensionless ratio $R / \sigma_{d}$; to understand the implications of equations 3 and 4 , we need only consider how $\Theta$ varies with this ratio, not the two variables independently.

This case is only of practical interest when the resistant form is rare, so the total proportion of the population killed is approximately the complement of the main parenthesis in equation 3 . Then, it is possible to examine how the change in the gene ratio, $\Theta$, depends on control and $\sigma_{d}$. Figure 2 shows a plot of $\Theta$, which measures the strength of selection, against $\sigma_{d} / R$ for various assumed values of the control when $u$ is 0 . Similar plots are obtained for all values of $u$, which shows that if the dose is adjusted to produce a particular level of kill, then the greater $\sigma_{d}$ is, slower the selection.

The control cannot exceed $1-u$. Below this level, selection varies with control, increasing up to an optimum value, after which the extra selection in the treated portion of the population does not compensate for the very small contribution it makes to the next generation compared with the sprayed portion (Fig. 3). The less variable are the spray doses are, the closer the maximum possible control is to the optimum.

Nonoverlapping generations in diploid, sexual organisms. In nonoverlapping generations of diploid, sexual organisms, there are three genotypes to consider. However, the important practical situation, while resistance is still relatively rare, is that only the susceptible homozygote, SS, and the resistant heterozygote, SR, are present at frequency $p$, which is close to 1 (as in equation 4 ), and $1-p$, respectively. If the allele frequency of $\mathrm{R}$ is $\phi$, then, assuming Hardy-Weinberg equilibrium, $1-p \approx 2 \phi$. The resistant homozygote will be very rare, and the dynamics of selection will be dominated by the fitness of the resistant heterozygotes. In this case, phenotypic selection can still be calculated as in

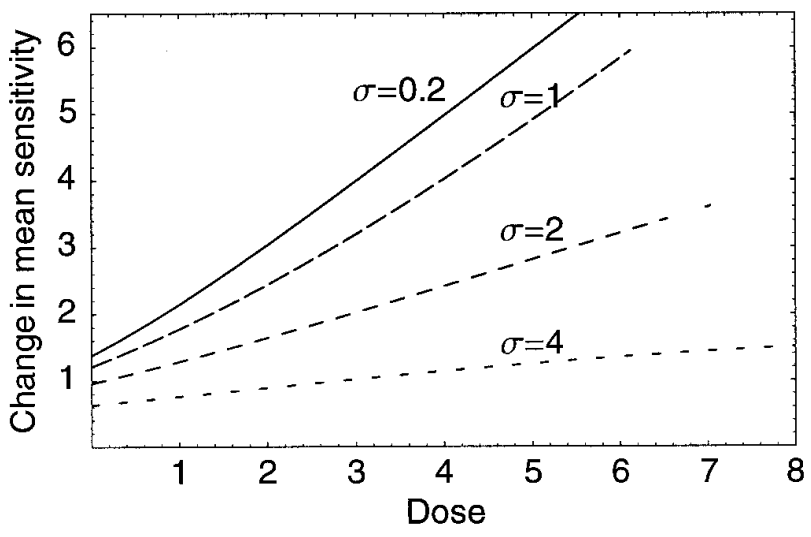

Fig. 4. Relation between the change in mean sensitivity, $\Delta$, and dose, without escape, for various values of standard deviation in dose received, $\sigma_{d}$. Both dose and sensitivity are measured in units of standard deviation of sensitivity. 
equations 3 and 4 . The new gene frequency is approximately half the new phenotype frequency. Genotype frequency and gene frequency changes are proportional, while the resistance remains rare, so the qualitative conclusions illustrated in Figures 2 and 3 still apply.

Polygenic resistance in clonal or haploid organisms. Nonoverlapping generations. The effect of selection on the mean value of sensitivity, $s$, after one generation can be calculated as follows. The survival probability of each phenotype, calculated by equation 1 , is multiplied by the proportion of the population consisting of that phenotype to find the new probability density function for sensitivity after treatment. The mean value of the sensitivity then can be found by integration.

In the following, the initial distribution of sensitivity is assumed to be described by equation 2. Analysis of the consequences of changes in dose and variability in dose is much simpler if dose is measured in units of the standard deviation in $s$, and if the initial value of average sensitivity is subtracted from all measurements, so that the new initial average sensitivity becomes 0 . With these simplifications, differentiating equation 2 gives the initial probability density of sensitivity:

$$
p(s)=\left[e^{\mathrm{s}} /\left(1+e^{\mathrm{s}}\right)^{2}\right]
$$

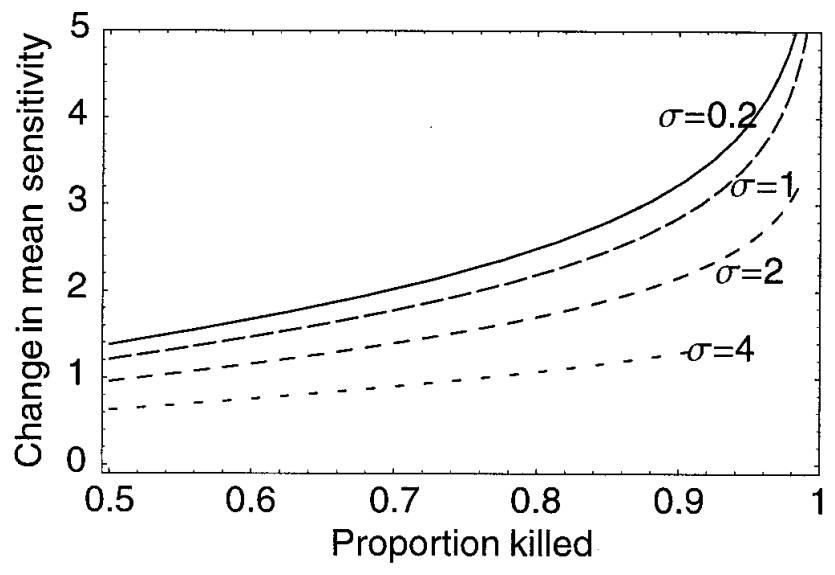

Fig. 5. Relation between change in mean sensitivity, $\Delta$, and level of control for various values of standard deviation in dose received, $\sigma_{d}$, without escape. Dose is adjusted to give the required degree of control, and the resulting $\Delta$ is plotted against the control. Sensitivity is measured in units such that its standard deviation is 1 .

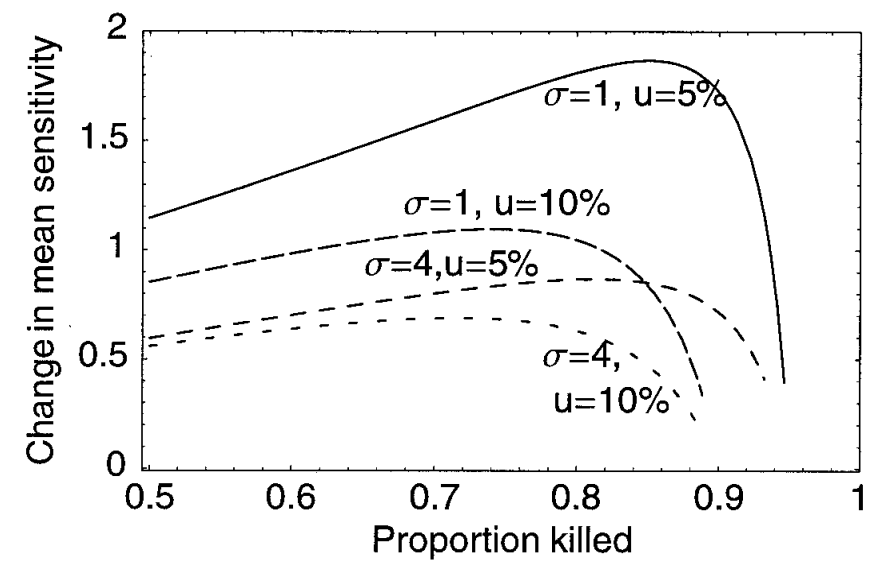

Fig. 6. The effect of escape on the relation between change in mean sensitivity, $\Delta$, and level of control for various values of standard deviation in dose received, $\sigma_{d}$, and escape. Dose is adjusted to give the required degree of control, and the resulting $\Delta$ is plotted against the control. Sensitivity is measured in units such that its standard deviation is 1 . and the change in sensitivity after treatment is

$$
\Delta=\frac{(1-u)}{(1-K)} \int_{-\infty}^{\infty} s \frac{e^{s}}{\left(1+e^{s}\right)^{2}} \frac{1}{1+e^{-\frac{\pi}{\sqrt{3}} \frac{\left(s-\mu_{d}\right)}{\sigma_{d}}}} d s
$$

with $1-K$ as the total proportion of survivors:

$$
1-K=u+(1-u) \int_{-\infty}^{\infty} \frac{e^{s}}{\left(1+e^{s}\right)^{2}} \frac{1}{1+e^{-\frac{\pi}{\sqrt{3}} \frac{\left(s-\mu_{d}\right)}{\sigma_{d}}}} d s
$$

Equation 6 describes the increase in resistance consequent to a single spray acting on an otherwise static population. Equation 7 describes the control resulting from the same spray. The integrations cannot be done analytically, and the equations must be studied numerically.

There are three independent variables, $u, \sigma_{d}$, and $\mu_{d}$. It is useful to examine first the case in which $u=0$. Figure 4 shows how the change in mean sensitivity, $\Delta$, varies with dose for various values of $\sigma_{d}$. With little variance in applied dose, a rising mean dose rapidly increases the change in mean sensitivity induced. For a larger variance, the same dose induces less change. Furthermore, as variance increases, $\Delta$ increases less rapidly with increased dose.

These graphs are potentially misleading, however, because with increased variance in dose, a given mean dose produces less control. In practice, dose is usually manipulated to produce adequate control. If in a particular agroecosystem $u$ and $\sigma_{d}$ were such that

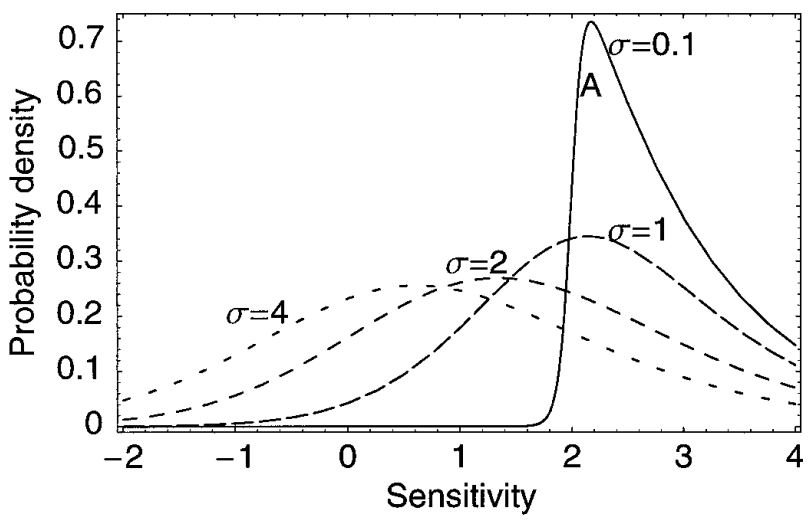

Fig. 7. Shape of frequency distribution of sensitivity after application of fungicide to a population with polygenically distributed resistance, for various values of the standard deviation in dose received, $\sigma_{d}$. The dose applied was 2 , on a scale such that the standard deviation of sensitivity is 1 , and the mean sensitivity is 0 .

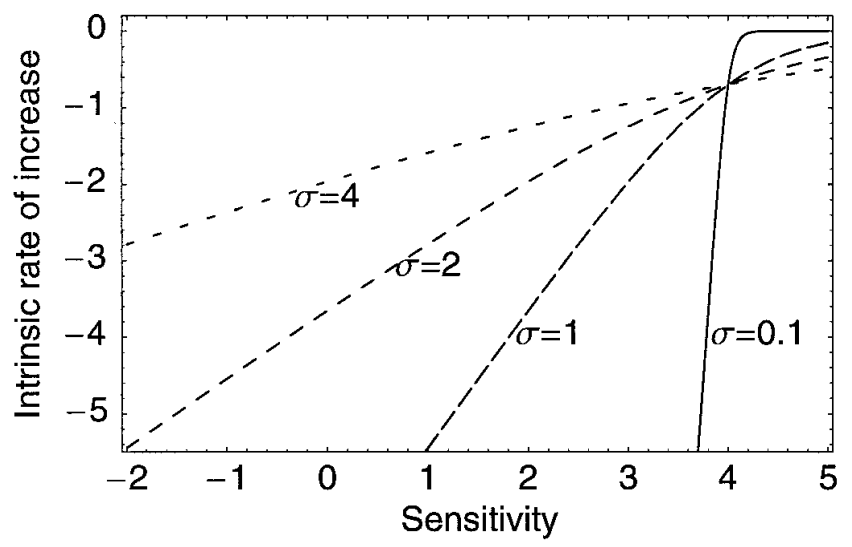

Fig. 8. Relation between resistance of a clone and its intrinsic rate of increase in continuous time, according to equation 8 , for different values of the standard deviation in dose received, $\sigma_{d}$. 
control was inadequate, doses would be adjusted, if possible, to improve control. Therefore, it is interesting to regard equations 6 and 7 as defining a relationship between level of control, $K$, and change in sensitivity, $\Delta$, parameterized by $u$ and $\sigma_{d}$. This relationship is shown in Figure 5. It is clear that the larger the variance in applied dose, the smaller the change in sensitivity for a given level of control, i.e., evolution of resistance will be slowest if the required control is achieved with maximum unevenness of pesticide application. However, the larger the unevenness, the larger the dose required for a given degree of control.

If $u$ is not 0 , a maximum appears in the graph of change in sensitivity against kill (Fig. 6), i.e., in a given agricultural setting, there is a critical dose at which sensitivity evolves most rapidly. If the doses used are greater than the critical dose, then increasing the dose will reduce the rate at which sensitivity evolves, but if the doses are typically less than the critical dose, then reducing the dose will reduce the rate. The problem in a practical setting is that it is likely to be impossible to find out whether current doses are more or less than the optimum.

The distribution of log sensitivity after a spray will no longer be logistic (Fig. 7). If an organism reproduces sexually, a moreor-less logistic distribution probably would be restored fairly rapidly, with a mean sensitivity possibly slightly reduced from that immediately after treatment, depending on heritability and the genetic architecture of the resistance. Indeed, one way of regarding increased variance in dose applied is as increased environmental variance in resistance, reducing the heritability of the resistance.

In an asexually reproducing organism, it is useful to begin by thinking about doses applied with little spread and no escape. In this case, only individuals surviving the dose applied would survive the first selection episode, and the population thereafter would be completely resistant, unless the dose were increased (Fig. 7, line A). With escape, the population can be thought of as a mixture of the original population and the completely selected one. The probability of an isolate escaping successive rounds of application will decline as the number of applications increases, and the distribution will converge exponentially, approximately, to the completely resistant case of Figure 7, line A. If the applied dose is heterogeneous, then the changes in response to subsequent applications of pesticide depend on how robust the description, so far, is when the assumptions of a logistically distributed sensitivity and dose are relaxed. This is discussed later.

Continuous time. One way to model these processes in continuous time is to assume the results of the previous section do not refer to living or dying but to reproduction as a whole. That is, if the average number of offspring, $R_{0}$, of a clone with resistance $s$

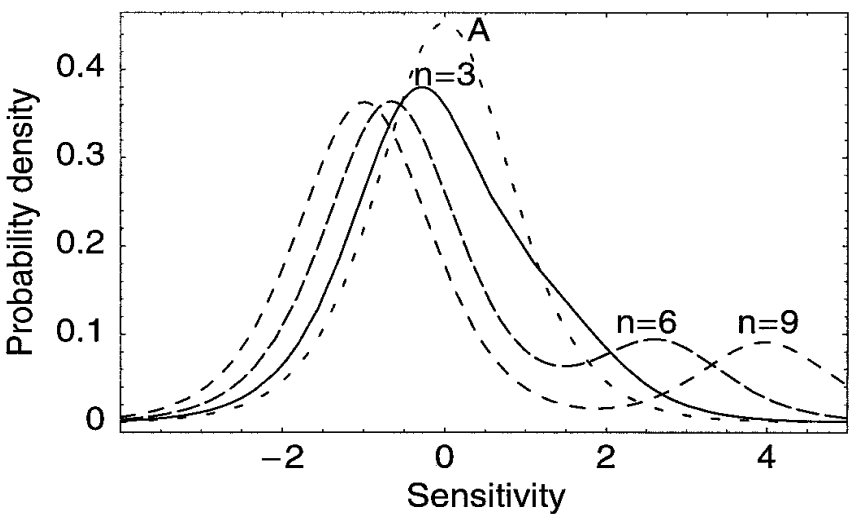

Fig. 9. Sample probability densities of dose according to equations 9 and 10 . All lines have $\mu$ (mean dose $)=0$ and $\sigma_{d}$ (standard deviation in dose $)=1$. Line $\mathrm{A}$ is a logistic curve, for reference. For all curves, $\lambda=0.8$; values of $<0.5$ would produce skew in the other direction. depended on the applied dose, according to equation 2 (with $u=$ $0)$, then the approximation

$$
r=\left\{\left[\ln \left(R_{0}\right)\right] / p\right\}
$$

implies that $r$, the intrinsic rate of increase, is proportional to the logarithm of equation 2 (with $u=0$ ). Figure 8 shows the result: a graph of the intrinsic rate of increase in the presence of fungicide against resistance level is more or less a straight line (justifying the assumption made in Shaw [20] that this was approximately true). The effect of increasing variance in dose is to reduce the slope of the line. Shaw (20) shows that, given the straight line relationship, the flatter this line is the more slowly resistance will be selected, independent of the intercept of the line with the resistance axis (which is determined by dose). This argument confirms the results of the previous section.

Sexual reproduction. Only the proportion of the variance in $s$ that is additively inherited is passed on. This proportion is known as the heritability, $h^{2}$ (5). However, this proportion does not affect the intensity of selection; it simply reduces the rate of selection to the fraction $h^{2}$ of the value deduced in equation 6. All the qualitative conclusions are unaffected. The presence of a sexual process also will restore approximate normality to the distribution of sensitivity in each generation, so the qualitative features of Figure 6 and equations 6 and 7 apply to both one and many generations.

Sensitivity of deductions to true form of dose or sensitivity distribution. The calculations in the case of polygenically inherited resistance were repeated with a dose distribution that was a mixture of two logistics combined so the mean and variance
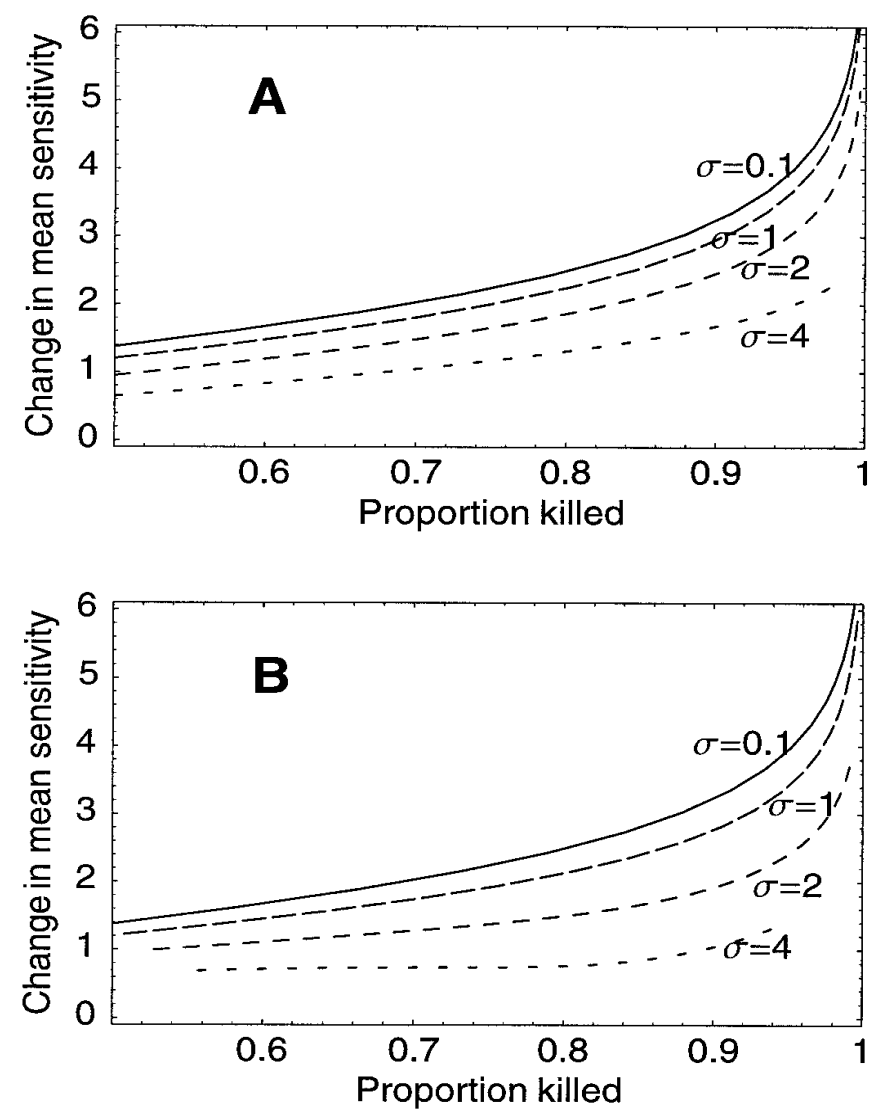

Fig. 10. The relation between extent of control and change in the mean resistance level of the population for asymmetric and bimodal dose distributions, with different standard deviations in dose, $\sigma_{d}$. The graphs are constructed as in Figure 5. A, For right-skewed distributions, $n=5$ and $\lambda=0.8$. B, For leftskewed distributions, $n=5$ and $\lambda=0.2$. 
could be held constant while the degree of asymmetry and bimodality were independently varied. The probability distribution function was given by

$$
P(X \leq x)=1-\left(\frac{\lambda}{1+e^{[x-(\mu+\beta n \lambda)] / \beta}}+\frac{(1-\lambda)}{1+e^{\{x-[\mu-\beta n(1-\lambda)] / / \beta}}\right)
$$

with

$$
\beta=\frac{\sigma}{\pi} \sqrt{\frac{3}{1+\left[3 n^{2} \lambda(1-\lambda)\right] / \pi^{2}}}
$$

In this distribution, as $n$ increases, the distribution becomes wider and more distinctly bimodal; as $\lambda$ varies from 0.5 , the distribution becomes more and more asymmetric (Fig. 9).

For the polygenic case, calculations equivalent to those leading to the result shown in Figure 5 are shown in Figure 10 for distributions that are distinctly bimodal and asymmetric either to the left (Fig. 10A) or right (Fig. 10B). It is clear that the qualitative conclusions drawn above are unaltered by large deviations from the assumptions about dose distribution. A normal distribution also was substituted in the calculations without altering the qualitative conclusions.

In the monogenic case, the logistic distributions in equations 3 and 4 can be substituted by general functions $F_{S}\left(\mu_{d} ; \sigma_{d}\right)$ for sensitive and $F_{R}\left(\mu_{d} ; \sigma_{d}\right)$ for resistant. If the expression for $\Theta$ is differentiated with respect to $\sigma_{d}$, it follows immediately that selection (measured by $\Theta$ ) will decrease with increasing variance in dose (measured by $\sigma_{d}$ ), provided only that $F_{R}\left(\mu_{d}\right)$ is always greater than
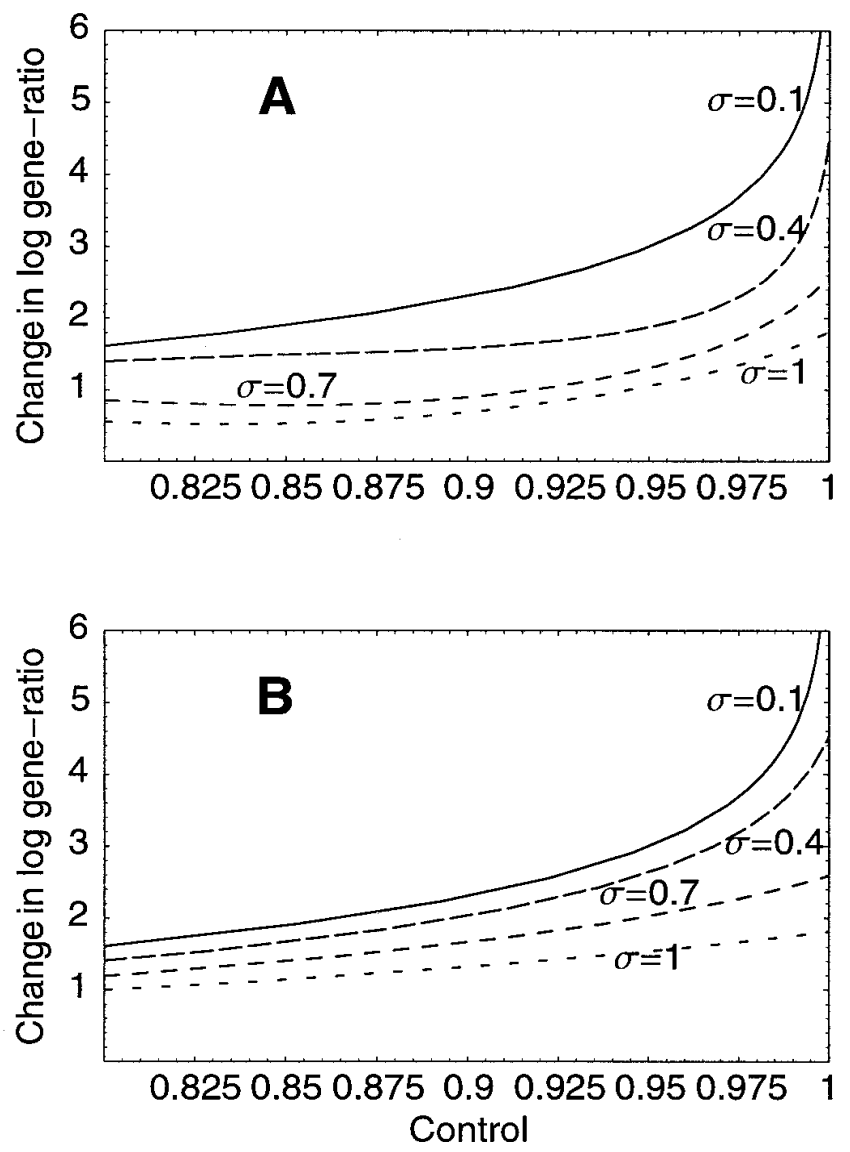

Fig. 11. The relation between extent of control and change in the log gene ratio of the population for asymmetric and bimodal dose distributions, with different standard deviations in dose, $\sigma_{d}$. The graphs are constructed as in Figure 3, except that escape is set to 0 in all cases. A, For right-skewed distributions, $n=5$ and $\lambda=0.8$. B, For left-skewed distributions, $n=5$ and $\lambda=0.2$.
$F_{S}\left(\mu_{d}\right)$. Because this is the criterion for regarding an isolate as resistant, it must be true for the range of doses used in the field, and the conclusion is completely general. $\left(F_{R}\right.$ need not be greater than $F_{S}$ over the whole range of possible doses (examples can be found in Metcalfe et al. [17].) An explicit example using the same bimodal distribution used in the previous case is shown in Figure 11.

\section{DISCUSSION}

The conclusions that can be drawn from the previous sections are that (i) the presence of escape produces a maximum in the graph relating selection to dose, and (ii) the greater the spread of dose experienced by treated individuals in the population for a given degree of control, the less selection occurs, and the more slowly selection increases with dose. These conclusions apply to asexual and sexual populations irrespective of the mode of inheritance of pesticide resistance or of the model of reproduction used. The conclusions also are insensitive to the exact form of dose distribution assumed.

The conclusions contradict the widely received wisdom about good use of pesticides, which is to minimize unintentional variation in dose arising from worn equipment and the uneven nature of the target (1). These standard recommendations will minimize the amount of pesticide required to achieve a given degree of control, but my paper suggests that they also will maximize the extent of selection for resistance.

The side-effects of pesticides must be related, in part, to the total dose applied, but it is not obvious how they are affected by heterogeneity in applied dose. The effect of dose heterogeneity must depend on the environmental effect considered. For example, poor spraying leading to larger doses must be more ecologically damaging if the damage occurs after the pesticide is volatilized or enters the groundwater, so the effect depends on the total concentration in the environment. On the other hand, some ecosystem side-effects that depend on the original spray pattern, such as those on coexisting beneficial organisms, may be minimized by a more variable spray and be less dependent on average dose. Some side-effects also may be closely correlated with the target effects and, as such, independent of mean dose. It is likely that no general conclusion applies, and it would be unfortunate to use the major conclusions of this paper to condone inefficient spraying in general.

The existence of a dose at which selection is maximal has not been explicitly argued before. Industry recommendations have always been to use only the recommended doses, and this has usually been stated as an antiresistance strategy (1). Currently, there is insufficient data (at least in the public domain) on most pesticides to know whether selection will be increased or decreased by altered doses. However, it is a common farming practice to reduce doses of some pesticides when pest pressure is perceived to be low. Steva (22) found increased selection of Uncinula necator on grape after reduced single doses of fungicide. Metcalfe et al. (17) found in a field experiment with a fungal pathogen of wheat, Мycosphaerella graminicola, that selection for resistance to the triazole fungicide fluquinconazole was reduced when doses were reduced from $1 / 4$ to $1 / 8$ of the recommended dose; however, this does not answer the question of where in relation to the maximum selection the recommended dose lies, and the necessary experiments would require considerable resources.

It is important to note that a reduced dose is relative to the effect on the target population. Applying the same amount of compound as in multiple sprays to achieve better control constitutes an increased dose or reduced variation in dose received for the purposes of the arguments presented above. For example, the experiments reported by Schulz (19) compared multiple reduced dose sprays with fewer higher dose sprays. This was appropriate as a test of a full system but provides no information about the effect of the individual sprays on selection. 
The analysis in my paper provides no support for the idea that there are intrinsic differences in the dose sensitivity of selection for monogenically and polygenically controlled resistance.

Gardner et al. (7) discussed a method of reducing the selection pressure for resistance by alternating high and low doses of pesticide. They did not allow for the effects of dose variation within a single application, however. Although the effect of this heterogeneity in time is in some ways similar to that discussed above, the mechanism is different, because it depends on periodic complete kill of the population, which may not be realistic for all organisms (Gardner et al.'s [7] examples were of weed populations). The underlying biology is similar to that which I argue will produce a maximum in the dose-selection curve and depends on being able to identify the position of that maximum and apply a dose much greater than the maximum. This is a slight generalization of Gardner et al.'s (7) results; it is not in fact necessary that kill be complete for their strategy to work, only that doses above those causing maximum change be used.

It obviously would be desirable to test the predictions of the models presented here in as many model systems as possible. It also might be desirable to undertake some modeling and experimental work to find out how the ecological consequences of pesticide use vary if the control is maintained constant but the dose is raised and deliberately made more variable.

These models also may have implications for deployment of pesticidal compounds as resistance factors constitutively or facultatively synthesized in genetically modified crops. Such a method of using a pesticidal compound is likely to lead to less variable doses than sprays or drenches, as well as reduced escape. This suggests that the risk of resistance will be raised using this method of deployment. Given the investment required to produce such a crop this possibility requires careful study.

\section{LITERATURE CITED}

1. Brent, K. J. 1995. Fungicide resistance in crop pathogens: How can it be managed? Groupement International des Associations Nationales de Fabricants de Produits Agrochimiques, Brussels.

2. Comins, H. N. 1986. Tactics for resistance management using multiple pesticides. Agric. Ecosyst. Environ. 16:129-148.

3. Cross, J. V., and Berrie, A. M. 1995. Field evaluation of a tunnel sprayer and effect of spray volume at constant drop size on spray deposits and efficacy of disease control on apple. Ann. Appl. Biol. 127:521-532.

4. Curtis, C. F., and Otoo, L. N. 1986. A simple model of the build-up of resistance to mixtures of anti-malarial drugs. Trans. Roy. Soc. Trop. Med. Hyg. 80:889-892.

5. Falconer, D. S., and Mackay, T. F. C. 1996. Introduction to Quantitative Genetics, 4th ed. Longman, Harlow, U.K.

6. Gan-Mor, S., Grinstein, A., Beres, H., Riven, Y., and Zur, I. 1996. Improved uniformity of spray deposition in a dense plant canopy: Methods and equipment. Phytoparasitica 24:57-67.

7. Gardner, S. N., Gressel, J., and Mangelm, M. 1998. A revolving dose strategy to delay the evolution of both quantitative vs major monogene resistances to pesticides and drugs. Int. J. Pest Man. 44:161-180.

8. Jagers op Akkerhuis, G. A. J. M., Axelsen, J. A., and Kjær, C. 1998. Towards predicting pesticide deposition from plant phenology: A study in spring barley. Pest. Sci. 53:252-262.

9. Kable, P. F., and Jeffrey, H. 1980. Selection for tolerance in organisms exposed to sprays of biocide mixtures: A theoretical analysis. Phytopathology 70:8-12.

10. Koch, H., and Spieles, M. 1992. Verteilung der Belagsmassen von Pflanzenschutzmitteln und Mehltaubefall (Erysiphe graminis f. sp. tritici) in einem Winterweizenbestand. Z. Pflanzenkr. Pflanzenschutz 99:297-303.

11. Koch, H., and Weisser, P. 1994. Untersuchungen zur Variabilität von Initialbelägen bei der Applikation von Pflanzenschutzmitteln in Obstanlagen. Z. Pflanzenkr. Pflanzenschutz 101:634-640.

12. Levy, Y., Levi, R., and Cohen, Y. 1983. Buildup of a pathogen subpopulation resistant to a systemic fungicide under various control strategies: A flexible simulation model. Phytopathology 73:1475-1480.

13. Mallet, J., and Luttrell, R. 1991. A model of insecticidal control failure: The example of Heliothis virescens on cotton. Southwest. Entomol. Suppl. 15:201-212.

14. Mani, G. S. 1985. Evolution of resistance in the presence of two insecticides. Genetics 109:761-783.

15. Mani, G. S. 1989. Evolution of resistance with sequential application of insecticides in time and space. Proc. Roy. Soc. B 238:245-276.

16. Mani, G. S., and Wood, R. J. 1984. Persistence and frequency of application of an insecticide in relation to the rate of evolution of resistance. Pest. Sci. 15:325-337.

17. Metcalfe, R. J., Shaw, M. W., and Russell, P. E. 1998. Factors affecting the strength of selection for resistance to DMI fungicides in Septoria tritici. Pages 535-542 in: Brighton 1998 Conf.: Pests Dis. British Crop Protection Council, Farnham, U.K.

18. Risch, S. J., Andow, D., and Altieri, M. A. 1983. Agroecosystem diversity and pest control: Data, tentative conclusions, and new research directions. Environ. Entomol. 12:625-629.

19. Schulz, U. 1994. Evaluating anti-resistance strategies for control of Erysiphe graminis f. sp. tritici. Pages 55-58 in: Fungicide Resistance. S. P. Heaney, D. Slawson, D. W. Hollomon, M. Smith, P. E. Russell, and D. W. Parry eds. British Crop Protection Council, Farnham, U.K.

20. Shaw, M. W. 1989. A model of the evolution of polygenically controlled fungicide resistance. Plant Pathol. 38:44-55.

21. Skylakakis, G. 1985. Two different processes for the selection of fungicideresistant subpopulations. EPPO Bull. 15:519-525.

22. Steva, H. 1994. Evaluating anti-resistance strategies for control of Uncinula necator. Pages 59-66 in: Fungicide Resistance. S. P. Heaney, D. Slawson, D. W. Hollomon, M. Smith, P. E. Russell, and D. W. Parry, eds. British Crop Protection Council, Farnham, U.K.

23. Via, S. 1985. Quantitative genetic models of evolution: Application to the control of pesticide resistance. Pages 222-235 in: Pesticide Resistance: Strategies and tactics for management. E. C. Cruze, ed. National Academy Press, Washington, DC.

24. Wood, R. J., and Mani, G. S. 1981. The effective dominance of resistance genes in relation to the evolution of resistance. Pest. Sci. 12:573-581. 